\title{
Joint Function and Arthropathy Severity in Patients with Hemophilia
}

\author{
Miwa Goto, $\mathrm{PT}, \mathrm{PhD}^{1 \#}$, Hideyuki Takedani, $\mathrm{MD}, \mathrm{PhD}^{2}$, Osamu NitTa, PT, $\mathrm{PhD}^{3}$ and \\ Kennosuke KaWAMA, PhD ${ }^{4}$ \\ 1) The University of Tokyo Hospital \\ 2) IMSUT Hospital of the Institute of Medical Science, The University of Tokyo \\ 3) Tokyo Metropolitan University \\ 4) University of Tsukuba
}

\begin{abstract}
Background: The Arnold-Hilgartner classification is one of the most popular evaluation systems for the progression hemophilic arthropathy. A previous study reported an association between arthropathy severity and arc range of motion (ROM). However, associations between arthropathy severity and angular ROM and muscle strength remain unclear. AIM: The purpose of this study was to clarify the association between joint function and arthropathy severity in hemophilia. Methods: We studied the knee, ankle, and elbow joints of 31 patients with hemophilia (PWH). The condition of the affected joints was evaluated on the basis of the interview data, joint function measurements, and roentgenography of the affected joints. In assessment of joint function, we evaluated knee strength (flexor, extensor) and grip strength as well as the passive ROM of the elbow, knee, and ankle. During the interview, all patients were asked about the history of intra-articular bleeding over the past year and pain. Results: As arthropathy severity worsened, knee flexor strength, knee extensor strength, grip strength, and ROM (elbow flexion, elbow extension, knee flexion, knee extension, and ankle extension) significantly decreased. Even patients with mild arthropathies experienced knee extensor weakness and extension limitation. In addition, joint function of severe ankle arthropathy was significantly related to the history of intra-articular bleeding and pain. Conclusion: Our results suggest that physical therapy is necessary to improve joint function in PWH and mild or no arthropathy. Pain control and prophylactic hematological management are necessary for patients with severe arthropathy because intra-articular bleeding and pain significantly decrease joint function.
\end{abstract}

Key words: Range of motion, Muscle strength, Arnold-Hilgartner classification

(J Jpn Phys Ther Assoc 18: 15-22, 2015)

Intra-articular bleeding is the most common manifestation of severe hemophilia. Following intra-articular bleeding, the joint becomes inflamed, tense, and warm, and the skin becomes bright red. Patients experience intense pain and hold the affected joint in flexion to decrease this pain ${ }^{1)}$. Repeated intra-articular bleeding episodes cause severe pain, diminish the range of motion (ROM) of affected joints, and loss of joint function and disability ${ }^{2}$. In addition, recurrent intra-articular bleeding also leads to synovial hy-

Received: January 7, 2015

Accepted: July 27, 2015

Advance Publication by J-STAGE: September 18, 2015

Correspondence to: Miwa Goto, The University of Tokyo Hospital,

7-3-1 Hongo, Bunkyo-ku, Tokyo 113-8655, Japan

\# e-mail: gotoum-reh@h.u-tokyo.ac.jp pertrophy and iron deposition (hemosiderin deposition) in the synovial membrane, resulting in chronic synovial inflammation $^{3)}$. These iron deposits initiate an inflammatory response and have a direct degenerative effect on cartilage ${ }^{4)}$. Repeated synovitis leads to progressive cartilage and subchondral bone destruction ${ }^{4}$. In a state of chronic synovitis, the joint may initiate a destructive process mediated by mechanical, chemical, and enzymatic mechanisms, which may result in irreversible damage ${ }^{5)}$. Degenerative joint damage and inflammatory processes occur in parallel from the early stages of the disease, resulting in a fibrotic destroyed joint with severe functional impairment ${ }^{6)}$. Hemophilic arthropathy (HA) causes major morbidity in patients with hemophilia $(\mathrm{PWH})^{7)}$, with most commonly affected 
joints being knees, ankles, and elbows ${ }^{8)}$. Thus, repeated intra-articular bleeding affects the progression of HA. In addition, pain caused by the intra-articular bleeding and HA significantly impacts activities of daily living and quality of life in $\mathrm{PWH}^{9,10)}$. The effects and safety of physiotherapy for hemophiliacs, included in end-stage arthropathy, has been reported $^{11,12)}$. However, the relationship between pain and arthropathy severity and intra-articular bleeding remains unclear, and a comprehensive assessment and clarification of this is important for physiotherapy. In particular, it is necessary to clarify how intra-articular bleeding and pain in end-stage arthropathy relates to joint function.

Previous studies have reported an association between HA severity and arc ROM, the sum of flexion ROM and extension $\mathrm{ROM}^{13)}$, but the relationship between $\mathrm{HA}$ and the angular ROM for each movement and muscle strength has not been reported. This study is the first report with a detailed assessment of the relationship between arthropathy severity, joint function, intra-articular bleeding, and pain in $\mathrm{PWH}$.

\section{Methods}

\section{Participants and methods}

This study included 31 PWH aged 16-61 years, selected from patients at a hospital registered for hemophilia in the Scientific and Standardization Committee of the Japanese Society on Thrombosis and Hemostasis. Patients with dysbasia and/or a history of orthopedic surgery within the previous month were excluded. We evaluated the condition of the patients' joints using the assessments of joint function, subject interview data, and roentgenographic assessments of the patients' joints. To assess joint function, we measured knee extensor strength, knee flexor strength, and grip strength, as well as the passive ROM of the elbow, knee, and ankle. Knee extensor and flexor strengths were measured using a hand-held dynamometer (microFET2; Hoggan Health Industries, Draper, UT, USA). Knee extensor and flexor strength were measured with the subject seated on a training table. The subjects were asked to droop the lower thigh. The training table pillar, distal portion of subjects' lower thigh, and sensor pad of a dynamometer were fixed using a belt. The subjects were then instructed to increase strength to maximal knee extension or flexion force and sustain this contraction for $3 \mathrm{~s} .{ }^{14)}$ Torque value (N $\times \mathrm{m}$ ) obtained by multiplying the maximum value (Newton) by lower thigh length (meter), which was divided by body weight $(\mathrm{kg})$, was recorded ${ }^{15}$. A Digital Grip Dynamometer (T.K.K.5401 Grip D. Takei Scientific Instruments Corp., Niigata, Japan) was used to measure bilateral grip strength. Subjects were asked to stand and the dynamometer was adjusted until the proximal interphalangeal joints were flexed to $90^{\circ}$ with the elbow straightened. The subjects were then instructed to increase pressure to maximal handgrip force and sustain the contraction for $3 \mathrm{~s}$. For consistency, the higher value of two measurements was used for the strength assessment based on methods of Kato et $a l .{ }^{14)}$ and Matsumura et al. ${ }^{16}$. Angular ROM was measured once in the supine position using a goniometer. The severity of arthropathy of the elbow, knee, and ankle was rated using the Arnold-Hilgartner classification, one of the most popular evaluation systems for the HA severity, on the basis of anteroposterior and lateral $\mathrm{x}$-rays obtained in the supine position ${ }^{17}$. During the interview, all subjects were asked about their hemostatic therapy, complications, intra-articular bleeding history over the previous year, and pain. The history of intra-articular bleeding over the previous year was evaluated by the presence or absence of bleeding in the subject joints. Similarly pain was evaluated by the presence or absence of pain in the subject joints. Data were collected regarding pain and intra-articular bleeding history of the elbow, knee, and ankle joints. Age, hemophilia severity, and complications were obtained from medical records.

\section{Ethical considerations}

This study was approved by the research ethics committees of the Faculty of Human Sciences at the University of Tsukuba and Institute of Medical Science, University of Tokyo. Written informed consent was obtained from all patients.

\section{Statistical analysis}

One-way analysis of variance (ANOVA) was used for analysis; ROM and strength were the dependent variables and arthropathy severity (Arnold-Hilgartner stages 0 to V) was the independent variable. Tukey's post hoc tests were used to compare arthropathy severity and joint function. Fisher's exact test was used to analyze differences in arthropathy severity (stage 0 to V) with intra-articular bleeding history, and also pain.

To allow comparisons between the subjects with severe arthropathy (stages IV or V), the subjects were divided into two groups for pain (those with pain and those without) and into two groups for intra-articular bleeding (those with bleeding and those without). Independent t-tests were then used to compare joint function between the two pain groups and between the two intra-articular bleeding groups.

SPSS 22.0J statistical software (IBM Japan Inc., Tokyo, Japan) was used for the analysis; statistical significance was defined as a $p$-value $<0.05$.

\section{Results}

\section{Baseline characteristics}

The study included 31 male subjects with a mean age of 38.3 years (range, 16-61 years). In total, 186 joints (right and left elbow, knee, and ankle joints) were assessed in each subject. However, ROM and grip strength were measured 
Table 1. ROM and arthropathy severity

\begin{tabular}{|c|c|c|c|c|c|c|c|c|c|c|c|}
\hline Joint & $\begin{array}{l}\text { Arnold } \\
\text { stage }\end{array}$ & $\mathrm{N}$ & $\begin{array}{c}\text { Mean } \\
\text { flexion } \\
\text { ROM } \\
\text { (degree) }\end{array}$ & S.D. & $P$ value & \multicolumn{2}{|c|}{$\begin{array}{c}\text { Multiple } \\
\text { comparison }\end{array}$} & $\begin{array}{c}\text { Mean } \\
\text { extension } \\
\text { ROM } \\
\text { (degree) }\end{array}$ & S.D. & $P$ value & $\begin{array}{c}\text { Multiple } \\
\text { comparison }\end{array}$ \\
\hline \multirow{6}{*}{ Elbow } & 0 & 5 & 136.0 & 2.2 & \multirow{6}{*}{$0.015^{*}$} & \multirow{6}{*}{\multicolumn{2}{|c|}{ n.s. }} & 0.0 & 0.0 & \multirow{6}{*}{$<0.001^{* * *}$} & $* * *$ \\
\hline & I & 8 & 135.0 & 0.0 & & & & -10.0 & 24.5 & & $* * *$ \\
\hline & III & 4 & 135.0 & 0.0 & & & & -2.5 & 5.0 & & $* *$ \\
\hline & IV & 7 & 126.4 & 6.9 & & & & -16.4 & 12.8 & & $*$ \\
\hline & V & 26 & 118.9 & 18.7 & & & & -40.4 & 16.6 & & \\
\hline & Total & 50 & 125.5 & 15.6 & & & & -25.1 & 22.9 & & \\
\hline & 0 & 10 & 139.5 & 2.8 & & $* * *$ & $* *$ & 0.0 & 0.0 & & 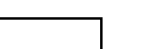 \\
\hline & I & 5 & 135.0 & 19.7 & & ** & $*$ & -2.0 & 4.5 & & \\
\hline & III & 5 & 139.0 & 2.2 & & ** & *** & -3.0 & 4.5 & & $*$ \\
\hline Knee & IV & 6 & 124.2 & 12.8 & $<0.001^{* * *}$ & $*$ & & -18.3 & 12.5 & $0.004^{* *}$ & \\
\hline & V & 16 & 83.8 & 41.3 & & & & -16.3 & 16.3 & & \\
\hline & TJA & 8 & 82.5 & 21.9 & & & & -8.1 & 9.6 & & \\
\hline & Total & 50 & 110.2 & 36.6 & & & & -9.2 & 12.9 & & \\
\hline & 0 & 2 & 35.0 & 7.1 & & & & 2.5 & 3.5 & & \\
\hline & I & 6 & 35.8 & 6.6 & & & & 12.5 & 5.2 & & \\
\hline Ankle & III & 6 & 30.8 & 3.8 & 012 & & S & 3.3 & 2.6 & $\cap \cap 01$ ** & $* * *$ \\
\hline 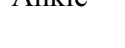 & IV & 14 & 33.2 & 6.4 & 0.12 & & . & 3.9 & 6.8 & 0.001 & Fan \\
\hline & V & 22 & 28.4 & 8.1 & & & & -1.8 & 7.5 & & \\
\hline & Total & 50 & 31.2 & 7.3 & & & & 2.3 & 7.8 & & \\
\hline
\end{tabular}

ROM: range of motion, S.D.: standard deviation, TJA: Post total joint arthoplasty, ${ }^{*}: \mathrm{p}<0.05,{ }^{* *}$ : $\mathrm{p}<0.01,{ }^{* * *}: \mathrm{p}<0.001$

ROM (excluding ankle flexion) decreased with arthropathy severity. Extension limitation appeared in early stage arthropathy but flexion limitation did not appear until the end stage of arthropathy for all three joints.

for only 25 patients ( 150 joints), because six subjects did not agree for ROM and grip strength measurement. Out of all subjects, $27(87.1 \%)$ had severe hemophilia and $4(12.9 \%)$ had moderate hemophilia; and $4(12.9 \%)$ had an inhibitor for defective coagulation factor. Twenty-two (71.0\%) subjects were treated prophylactically and $9(29.0 \%)$ were receiving on-demand treatment. Regarding viral infections, 24 (77.4\%) subjects had hepatitis C, 12 (38.7\%) had the human immunodeficiency virus, and $6(19.4 \%)$ had hepatitis $B$ virus.

\section{ROM and arthropathy severity (Table 1)}

For the elbow, one-way ANOVA showed statistically significant differences across the arthropathy severity stages in both flexion $\operatorname{ROM}[F(4,45)=3.46, p=0.015]$ and extension $\operatorname{ROM}[F(4,45)=12.60, p<0.001]$. Tukey's tests showed the following significant differences in extension ROM between the stages: between stage 0 and stage $\mathrm{V}(p<$ $0.001)$, stage I and stage $\mathrm{V}(p<0.001)$, stage III and stage $\mathrm{V}$ $(p=0.001)$, and between stage IV and stage $\mathrm{V}(p=0.011)$

For the knee, one-way ANOVA showed statistically significant differences across the arthropathy severity stages in both flexion $\operatorname{ROM}[F(5,44)=9.60, p<0.001]$ and extension ROM $[F(5,44)=4.12, p=0.004]$. Tukey's tests showed the following significant differences in flexion ROM between the stages: between stage 0 and stage $\mathrm{V}(p<$ $0.001)$, stage I and stage $\mathrm{V}(p=0.007)$, stage III and stage $\mathrm{V}$ $(p=0.003)$, and between stage IV and stage $\mathrm{V}(p=0.032)$. Knee flexor ROM in stages 0 , I, and III were significantly related to that in post total joint arthroplasty (TJA) ( $p=$ $0.001, p=0.015$, and $p=0.007$, respectively). Tukey's test showed a significant difference between the knee extension ROM in stage 0 and in stage $\mathrm{V}(p=0.035)$.

For the ankle, one-way ANOVA showed a statistically significant difference across the arthropathy severity stages in extension $\operatorname{ROM}[F(4,45)=5.95, p=0.001]$ but not in flexion ROM. Tukey's test showed that extension ROM in stage I was significantly different from that in stage $\mathrm{V}(p<$ $0.001)$. Extension limitation was apparent in the early stage of arthropathy but flexion limitation did not appear until the end stage of arthropathy for all three joints.

\section{Strength and arthropathy severity (Tables 2 and 3)}

One-way ANOVA showed a statistically significant difference in grip strength across the elbow arthropathy severity stages $[F(4,57)=3.57, p=0.012]$. Tukey's test showed 
Table 2. Grip strength and elbow arthropathy severity

\begin{tabular}{ccccccc}
\hline Joint & $\begin{array}{c}\text { Arnold } \\
\text { stage }\end{array}$ & $\mathrm{N}$ & $\begin{array}{c}\text { Mean grip } \\
\text { strength }(\mathrm{kg})\end{array}$ & S.D. & P value & $\begin{array}{c}\text { Multiple } \\
\text { comparison }\end{array}$ \\
\hline \multirow{5}{*}{ Elbow } & 0 & 5 & 38.5 & 9.5 & & \\
& I & 8 & 41.6 & 9.4 & & \\
& III & 4 & 37.5 & 9.5 & $0.012^{*}$ & \\
& IV & 7 & 39.1 & 10.9 & & \\
& V & 26 & 31.3 & 8.2 & & \\
\hline
\end{tabular}

S.D.: standard deviation, ${ }^{*}: \mathrm{p}<0.05$

Grip strength decreased with elbow arthropathy severity.

Table 3. Knee strength and knee arthropathy severity

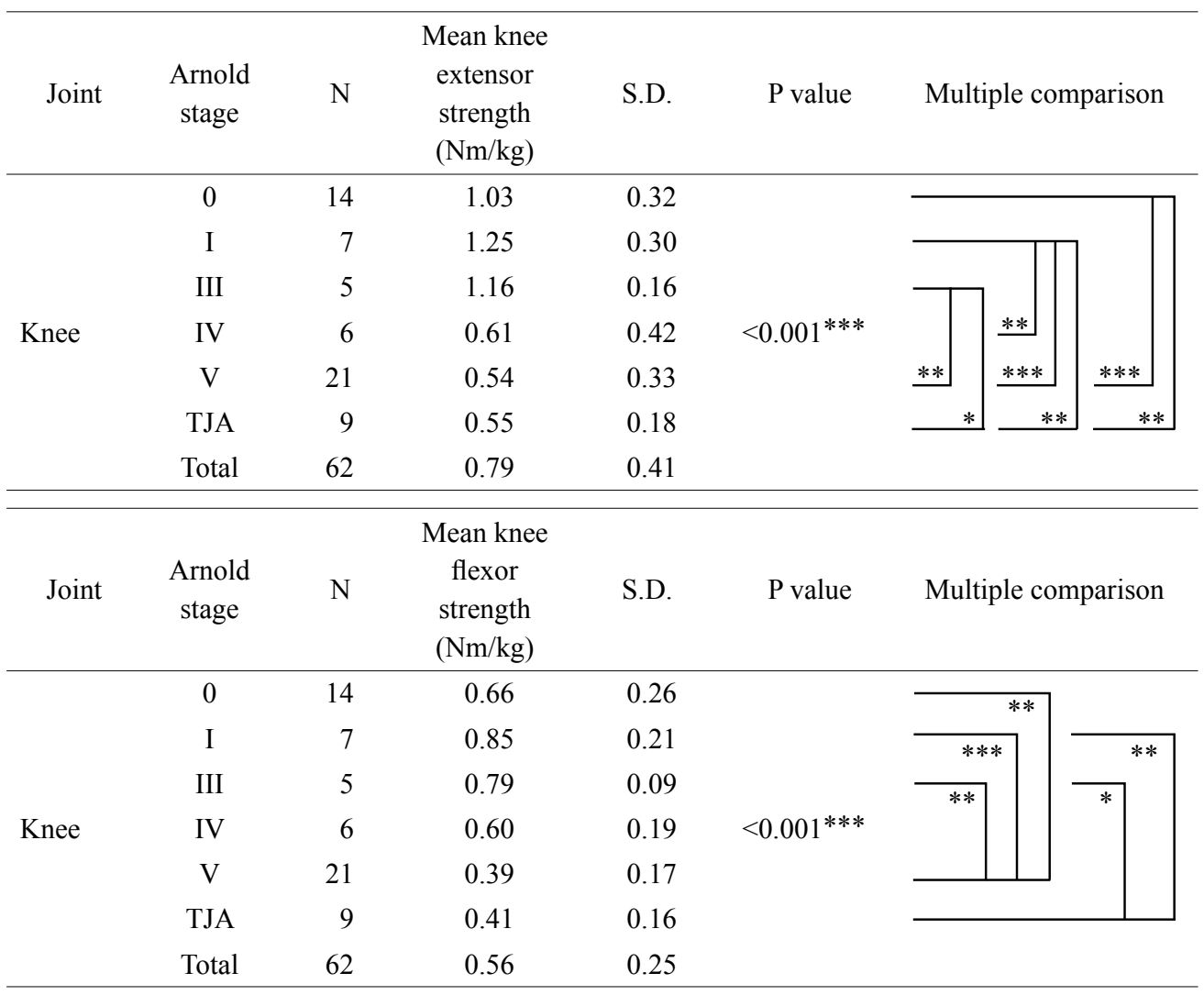

S.D.: standard deviation, TJA: Post total joint arthoplasty, ${ }^{*}$ : $p<0.05,{ }^{* *}: \mathrm{p}<0.01,{ }^{* * *}$ : $\mathrm{p}<0.001$

Knee strength decreased with arthropathy severity. Knee flexor and extensor strengths were decreased even at an early stage of arthropathy and were much worse at the end stage of arthropathy.

a significant difference between grip strength in stage I and in stage $\mathrm{V}(p=0.013)$. One-way ANOVA showed statistically significant differences across the knee arthropathy severity stages in knee extensor strength $[F(5,56)=10.42$, $p<0.001]$ and knee flexor strength $[F(5,56)=9.18, p<$ $0.001]$

Knee flexor and extensor strengths were reduced even at an early stage of arthropathy and were much worse by the end stage. Tukey's tests showed the following significant differences in knee extensor strength between stages: between stage I and stage IV $(p=0.006)$, between stage 0 and stage $\mathrm{V}(p<0.001)$, between stage I and stage $\mathrm{V}(p<$ $0.001)$, and between stage III and stage V $(p=0.002)$. Knee extensor strength in stages 0 , I, and III were significantly related to that in post total joint arthroplasty (TJA) ( $p=$ $0.008, p=0.001$, and $p=0.011$, respectively). The significant differences found for knee flexor strength between the stages were: between stage 0 and stage V $(p=0.004)$, between stage I and stage $\mathrm{V}(p<0.001)$, and between stage III and stage V $(p=0.002)$. Knee flexor strength in stages I and III were significantly related to that in TJA $(p=0.001$ and $p=0.012$, respectively). 
Table 4. Arthropathy severity, intra-articular bleeding, and pain

\begin{tabular}{|c|c|c|c|c|c|c|c|c|}
\hline \multirow{2}{*}{ Joint } & \multirow{2}{*}{ Physical findings } & \multicolumn{5}{|c|}{ Arnorld stage } & \multirow{2}{*}{ TJA } & \multirow{2}{*}{ P value } \\
\hline & & 0 & $\mathrm{I}$ & III & IV & $\mathrm{V}$ & & \\
\hline \multirow{4}{*}{ Elbow } & Without bleeding & 6 & 8 & 3 & 6 & 21 & 0 & \multirow{2}{*}{0.991} \\
\hline & With bleeding & 2 & 4 & 1 & 2 & 9 & 0 & \\
\hline & Without pain & 7 & 11 & 3 & 6 & 19 & 0 & \multirow{2}{*}{0.342} \\
\hline & With pain & 1 & 1 & 1 & 2 & 11 & 0 & \\
\hline \multirow{4}{*}{ Knee } & Without bleeding & 14 & 6 & 4 & 3 & 9 & 4 & \multirow{2}{*}{$0.007^{* *}$} \\
\hline & With bleeding & 0 & 1 & 1 & 3 & 12 & 5 & \\
\hline & Without pain & 14 & 7 & 4 & 2 & 6 & 5 & \multirow{2}{*}{$<0.001^{* * *}$} \\
\hline & With pain & 0 & 0 & 1 & 4 & 15 & 4 & \\
\hline \multirow{4}{*}{ Ankle } & Without bleeding & 1 & 5 & 2 & 14 & 15 & 0 & \multirow{2}{*}{0.247} \\
\hline & With bleeding & 1 & 2 & 4 & 4 & 14 & 0 & \\
\hline & Without pain & 1 & 4 & 2 & 12 & 15 & 0 & \multirow{2}{*}{0.684} \\
\hline & With pain & 1 & 3 & 4 & 6 & 14 & 0 & \\
\hline
\end{tabular}

TJA: Post total joint arthoplasty, ${ }^{* *}: \mathrm{p}<0.01,{ }^{* * *}: \mathrm{p}<0.001$

Arthropathy was significantly related to intra-articular bleeding history and pain at the knee joint.

Table 5. Joint function and intra-articular bleeding and pain in subjects with severe arthropathy

\begin{tabular}{|c|c|c|c|c|c|c|c|c|c|c|c|c|}
\hline \multirow{2}{*}{ Joint } & \multirow{2}{*}{$\mathrm{N}$} & \multirow{2}{*}{ Joint function } & \multicolumn{2}{|r|}{ With pain } & \multicolumn{2}{|c|}{ Without pain } & \multirow{2}{*}{$P$ value } & \multicolumn{2}{|c|}{ With bleeding } & \multicolumn{2}{|c|}{ Without bleeding } & \multirow{2}{*}{$P$ value } \\
\hline & & & $\mathrm{N}$ & Mean (S.D) & $\mathrm{N}$ & Mean (S.D) & & $\mathrm{N}$ & Mean (S.D) & $\mathrm{N}$ & Mean (S.D) & \\
\hline \multirow{3}{*}{ Elbow } & 33 & Flexion ROM (degree) & 12 & $122.5(8.4)$ & 21 & $119.3(20.6)$ & 0.612 & 10 & $123.0(9.2)$ & 23 & $119.4(19.7)$ & 0.581 \\
\hline & 33 & Extension ROM (degree) & 12 & $-36.3(14.9)$ & 21 & $-34.8(20.7)$ & 0.829 & 10 & $-37.0(19.5)$ & 23 & $-34.6(18.6)$ & 0.735 \\
\hline & 38 & Grip strength (kg) & 13 & $29.1(8.5)$ & 25 & $35.0(9.1)$ & 0.062 & 11 & $31.5(10.7)$ & 27 & $33.5(8.7)$ & 0.553 \\
\hline \multirow{4}{*}{ Knee } & 22 & Flexion ROM(degree) & 16 & $106.3(27.4)$ & 6 & $64.2(54.0)$ & 0.118 & 13 & $113.1(22.1)$ & 9 & $68.3(46.2)$ & $0.021^{*}$ \\
\hline & 22 & Extension ROM (degree) & 16 & $-16.9(15.7)$ & 6 & $-16.7(14.7)$ & 0.978 & 13 & $-19.6(17.6)$ & 9 & $-12.8(10.0)$ & 0.262 \\
\hline & 27 & Knee extensor strength $(\mathrm{Nm} / \mathrm{kg})$ & 19 & $0.52(0.34)$ & 8 & $0.64(0.37)$ & 0.392 & 15 & $0.56(0.36)$ & 12 & $0.55(0.33)$ & 0.943 \\
\hline & 27 & Knee flexor strength (Nm/kg) & 19 & $0.43(0.19)$ & 8 & $0.45(0.21)$ & 0.834 & 15 & $0.40(0.18)$ & 12 & $0.48(0.20)$ & 0.301 \\
\hline \multirow{2}{*}{ Ankle } & 36 & Flexion ROM (degree) & 14 & $26.1(8.1)$ & 22 & $33.0(6.3)$ & $0.007^{* *}$ & 14 & $26.4(8.9)$ & 22 & $32.7(5.9)$ & $0.015^{*}$ \\
\hline & 36 & Extension ROM (degree) & 14 & $1.8(5.8)$ & 22 & $-0.5(8.7)$ & 0.402 & 14 & $1.1(5.3)$ & 22 & $0.0(9.0)$ & 0.690 \\
\hline
\end{tabular}

ROM: range of motion, S.D.: standard deviation, TJA: Post total joint arthoplasty, ${ }^{*}$ : $\mathrm{p}<0.05,{ }^{* *}$ : $\mathrm{p}<0.01$

Ankle joint function differed with pain and intra-articular bleeding among subjects with severe ankle arthropathy.

Arthropathy severity, intra-articular bleeding, and pain (Table 4)

Fisher's exact test showed significant differences in knee arthropathy severity with intra-articular bleeding history $(p=0.007)$ and pain $(p<0.001)$. However, elbow arthropathy severity and ankle arthropathy severity did not differ significantly with intra-articular bleeding history or pain.

Joint function and intra-articular bleeding and pain in subjects with severe arthropathy (Table 5)

Comparisons were made of joint function in the subjects with severe arthropathy (stages IV and V) between the two pain groups and between the two intra-articular bleeding groups. Knee flexion ROM in those with intraarticular bleeding history was significantly higher than in those without bleeding $(p=0.021)$. Ankle flexion ROM in severe arthropathy subjects with pain was significantly lower than in those without pain $(p=0.007)$. Ankle flexion ROM in severe arthropathy subjects with a history intraarticular bleeding history was significantly lower than in those without bleeding ( $p=0.015)$.

\section{Discussion}

ROM and arthropathy severity

Previous studies have reported that joint ROM decreases with the progression of $\mathrm{HA}^{13)}$. Our study supports these findings; however, ankle flexion ROM did not show a decrease with HA severity progression. As HA progresses, bone spurs and cysts form and articular surfaces become irregular, eventually resulting in narrowing of the joint space $^{4)}$. Reduced ROM is, therefore, related to structural joint changes. However, the severe pain and joint swelling 
experienced after acute intra-articular bleeding of the ankle drive the ankle into an antalgic plantar flexion attitude ${ }^{18)}$. In addition, repetitive bleeding into the ankle will produce a marginal osteophyte on the anterior portion of the tibia in the ankle; this deformity results from a combination of the contracture of the posterior capsule of the ankle and retraction of the Achilles tendon and collapse of the dome of the talus, due to osteonecrosis and cartilage deterioration ${ }^{18)}$. Therefore, it seems that the relationship between ankle flexion ROM and arthropathy severity was not significant in this study.

Exceptionally, the elbow joints in some subjects in our study showed increased flexion ROM even at the end stage of arthropathy. As arthropathy progresses, the olecranon fossa expands, the humerus becomes fragile because of osteolysis, and joint instability increases, eventually resulting in fractures of the humerus ${ }^{19}$. This may be the reason why the Tukey's tests showed no significant differences in elbow flexion ROM with arthropathy severity progression. However, there were significant differences between knee flexion ROM at different stages of arthropathy severity. The remarkable knee flexion limitation observed in stage $\mathrm{V}$ may be due to a range of factors such as epiphyseal overgrowth, joint disorganization, squaring of the patella, widened notch of the distal femur, and severe degenerative changes in the patellofemoral and tibiofemoral compartments ${ }^{20)}$. It is necessary to assess arthropathy using roentgenography before physiotherapy interventions so that physiotherapy can be planned according to the severity of arthropathy.

In the subjects with mild arthropathy, all three joints showed limitations in extension ROM, except in those with stage I ankle arthropathy. Limitations in extension ROM were significant in stage IV and V. This result suggests that joints affected by intra-articular bleeding were held in antalgic flexion with painful and limited mobility. As a result, the soft tissue and muscles on the flexor aspect of the limb had shortened. In addition, features of advancing osteoarthritis can be seen radiologically in stage IV, and articular cartilage destruction is evidenced by the narrow joint space $^{20)}$. In stage $\mathrm{V}$, there are fibrous joint contractures, loss of the joint cartilage space, and extensive enlargement of the epiphyses with substantial disorganization of the joint $^{20)}$. Because of these reasons, it is believed, a significant limitation in extension ROM was observed in the end stage in our subjects. Passive mobilization and joint traction are physiotherapy techniques for maintaining ROM in $\mathrm{HA}^{21)}$. Cuesta-Barriuso reported that joint traction of the knee, ankle, and elbow improved ROM in $\mathrm{PWH}^{22)}$. Even for mild arthropathy, it has been suggested that passive mobilization, joint traction, and stretching are important for improving extension.

\section{Strength and arthropathy severity}

Quadriceps weakness is a pathological hallmark of se- vere hemophilia in adults ${ }^{23)}$. It has been reported that knee extensor strength in PWH is distinctly lower than in healthy individuals ${ }^{24-26)}$. It has also been reported that quadriceps weakness in PWH is present throughout the adult life and that the strength difference between these patients and healthy individuals widens with increasing age ${ }^{23)}$. However, no differences have been reported with regard to knee flexor strength between PWH and healthy individuals ${ }^{27}$. Between intra-articular bleeding episodes, PWH show no difference in knee flexor strength between the involved knee and the uninvolved knee ${ }^{28}$. However, although the knee is the joint most commonly affected by arthropathy and intra-articular bleeding, previous researches on knee muscle in hemophilia are restricted to only the reports cited here. The relationship between knee flexor strength and the knee arthropathy severity remains unclear.

Knee extensor and flexor strength decreased with knee arthropathy severity progression in this study. In addition, extensor strength was significantly weakened in stage IV arthropathy and flexor strength was significantly weakened in stage $\mathrm{V}$. It has been reported that patients with patellofemoral joint pain experience a dysfunction of the knee extension mechanism due to a delay in the vastus medialis reaction ${ }^{29)}$, and that increased intra-articular pressure induces reflective suppression of the quadriceps muscle in the full extension position of the knee for patients with hydrarthrosis ${ }^{29}$. In addition, anxiety due to pain deters quadriceps contraction, leading to muscle atrophy ${ }^{30}$. Thus, knee extensor strength is closely related to intra-articular pressure and patellofemoral joint pain. Knee extensor strength is also affected by the arthropathy severity and intra-articular bleeding. These reasons suggest that extensor muscles may weaken earlier than flexor muscles.

Even subjects with mild arthropathy showed knee extensor and flexor weakness in this study. Specifically, the knee extensor strength in stage 0 was weaker than the cut-off value for independence in walking and climbing stairs $(1.4 \mathrm{Nm} / \mathrm{kg})$ and fall risk value $(1.2 \mathrm{Nm} / \mathrm{kg})^{31)}$; it was also lower than that of the elderly healthy individuals ${ }^{32)}$. Even PWH with normal joints or mild arthropathy show decreased muscular strength and reduced physical activity $^{8)}$. In addition to its main function as a knee extensor, the quadriceps muscle also assists in controlling patellar tracking during knee flexion to prevent excessive lateral displacement ${ }^{33)}$. Muscle weakness around the joint, including flexor weakness, can lead to joint instability and increased risk of intra-articular rebleeding. Therefore, strength training is essential for knee extensors and flexors after control of intra-articular bleeding. Early physiotherapy intervention to prevent and treat muscle weakness and low physical activity is also necessary.

Grip strength significantly decreased with severity of elbow arthropathy in this study. Grip strength reflects the functional status of the upper extremity, and this measure 
has been used in clinical studies of upper extremity involvement ${ }^{34)}$. However, no previous reports have assessed grip strength together with hemophilic elbow arthropathy. Our results suggest that a detailed assessment and strength training for the upper extremities are necessary.

\section{Intra-articular bleeding, pain, and arthropathy severity}

In this study, severity of arthropathy was related to intra-articular bleeding history and pain at the knee joint, but not at the elbow or ankle joints. A discrepancy between intra-articular bleeding frequency and arthropathy severity has been reported in another study that evaluated joint function in $\mathrm{PWH}^{35)}$. It is clear severity of arthropathy is related with pain and with intra-articular bleeding, but the manifestation of this depends on the joint. One reason for this is that greater bleeding frequency is affected by synovitis state. Ultrasonography, palpation, and magnetic resonance imaging are useful and necessary for the diagnosis of synovitis ${ }^{36}$. Subchondral bone cysts, loss of the joint cartilage space, extensive enlargement of the epiphyses, and substantial disorganization of the joint are observed in knee arthropathy stages IV and V. In addition, there is also lateral subluxation of the tibia. Standing or walking applies a load to the knee joint. In particular severe knee arthropathy, it is presumed that chronic pain, intra-articular bleeding frequency, and subperiosteal bleeding frequency from cysts are increased by weight bearing. The ankle is also a weight-bearing joint. However, in severe ankle arthropathy there is retraction of the posterior ankle capsule, shortening of the Achilles tendon, and collapse of the dome of the talus, due to osteonecrosis and cartilage deterioration ${ }^{18)}$. Therefore, it may be the case that even subjects with severe ankle arthropathy have less chronic pain and intra-articular bleeding because the joint was almost ankylosed. Therefore, we believe that physiotherapy intervention is required for joints that have intra-articular bleeding history or pain, even in the absence of arthropathy.

\section{Joint function, intra-articular bleeding and pain in subjects with severe arthropathy}

Flexion ROM in subjects with severe ankle arthropathy without pain or intra-articular bleeding history was significantly higher than in those with intra-articular bleeding history or pain. This may be because ankle synovial hypertrophy and synovitis reduce the plantar flexion ROM even at the end stage of arthropathy, as ankle bleeding frequency increases. However, flexion ROM in subjects with severe knee arthropathy without intra-articular bleeding history was significantly lower than in those with intra-articular bleeding history. It is suggested that this result was affected by knee joint structure more than bleeding frequency. However, only arthropathy severity was evaluated in this study. This point should be studied further in future.

Pain has been described as the leading cause of dis- ability in $\mathrm{PWH}^{37)}$ and a previous study reported that physiotherapy provides pain relief in $\mathrm{PWH}^{22}$. Patients with severe arthropathy, therefore, require physiotherapy to improve joint function and pain, and should receive pain control and prophylactic hematological management.

\section{Conclusion}

The purpose of this study was to clarify the relationship between arthropathy severity and joint function in PWH. Assessments of joint function and arthropathy were performed for the elbow, knee, and ankle joints. Knee extensor strength, knee flexor strength, grip strength, and ROM (excluding ankle flexion) decreased with arthropathy severity. Muscle weakness and extension limitation were observed even in subjects with mild arthropathy suggesting that physiotherapy interventions to improve joint function may be necessary for PWH with mild or no arthropathy. Ankle joint function differed with pain and intra-articular bleeding among subjects with severe ankle arthropathy in this study. This suggests pain control and prophylactic hematological management are also necessary for patients with severe arthropathy. It is important to provide early physiotherapy intervention to improve joint function before arthropathy progresses.

\section{Reference}

1) Rodriguez-Merchan EC: Articular bleeding (hemarthrosis) in hemophilia. An orthopedist's point of view. $2^{\text {nd }}$ ed, Montréal, the World Federation of Hemophilia, Schulman S. 2008, pp. $1-5$.

2) Rodriguez-Merchan EC: Effects of hemophilia on articulations of children and adults. Clinical Orthopaedics and Related Research. 1996, 328: 7-13.

3) Valentino LA, Hakobyan N, and Enockson C: Blood-induced joint disease: The confluence of dysregulated oncogenes, inflammatory signals and angiogenic cues. Seminars in Hematology. 2008, 45: 50-57.

4) Cross S, Vaidya S, and Fotiadis N: Hemophilic arthropathy: a review of imaging and staging. Semin Ultrasound CT MR. 2013, 34(6): 516-524.

5) Mulder K, and Llinás A: The target joint. Haemophilia. 2004, 10(4): 152-156.

6) Roosendaal G, van Rinsum AC, Vianen ME, van den Berg HM, Lafeber FP, and Bijlsma JW: Haemophilic arthropathy resembles degenerative rather than inflammatory joint disease. Histopathology. 1999, 34 (2): 144-153.

7) Srivastava A, Brewer AK, Mauser-Bunschoten EP, Key NS, Kitchen S, Llinas A, Ludlam CA, Mahlangu JN, Mulder K, Poon MC, and Street A: Guidelines for the management of hemophilia. Haemophilia. 2013, 19(1): 1-47.

8) Gomis M, Querol F, Gallach JE, González LM, and Aznar JA: Exercise and sport in the treatment of haemophilic patients: a systematic review. Haemophilia. 2009, 15: 43-54.

9) Goto M, Takedani H, Kawama K, and Nitta O: Factors related to quality of life in patients with hemophilia. Jpn J Thromb 
Hemost. 2014, 25(3): 388-395. (in Japanese)

10) Molho P, Rolland N, Lebrun T, Dirat G, Courpied JP, Croughs T, Duprat I, and Sultan Y: Epidemiological survey of the orthopaedic status of severe haemophilia A and B patients in France. Haemophilia. 2000, 6(1): 23-32.

11) Greene WB, and Strickler EM: A modified isokinetic strengthening program for patients with severe hemophilia. Dev Med Child Neurol. 1983, 25: 189-196.

12) Goto $M$, Takedani $H$, Haga $N$, Kubota $M$, Ishiyama $M$, Ito $S$, and Nitta O: Self-monitoring has potential for home exercise programs in patients with hemophilia. Haemophilia. 2014, 20: e121-e127.

13) Johonson RP, and Babbitt DP: Five stages of joint disintegration compared with range of motion in hemophilia. Clinical Orthopaedics. 1985, 201: 36-42.

14) Kato M: Measurements of Isometric Knee Extension Force with a Hand-Held Dynamometer: Influence which the Difference in the Setting Method has on Measured value. The Journal of Tokyo Academy of Health Science. 2003, 6(3): 201-204. (in Japanese)

15) Whiteley R, Jacobsen P, Prior S, Skazalski C, Otten R, and Johnson A: Correlation of isokinetic and novel hand-held dynamometry measures of knee flexion and extension strength testing. J Sci Med Sport. 2012, 15(5): 444-450.

16) Matsumura $M$, Takei $H$, Ichikawa $K$, Ogawa $D$, Usa $H$, and Hata M: The inter- and intra-rater reliability of the isometric muscle strength measurement by belt fixed hand-held dynamometer: The measurements of knee flexors, ankle dorsiflexors, ankle plantar flexors, ankle evertors, ankle invertors. The Journal of Japan Academy of Health Science. 2012, 15(1): 41-47. (in Japanese)

17) Arnold WD, and Hilgartner MW: Hemophilic arthropathy. Current concepts of pathogenesis and management. J Bone Joint Surg. 1977, 59: 287-305.

18) Llinás A: The ankle joint. Haemophilia. 2010, 16(5): 124-125.

19) Gamble JG, Vallier H, Rossi M, and Glader B: Loss of elbow and wrist motion in hemophilia. Clin Orthop Relat Res. 1996, 328: 94-101.

20) Handelsman JE: The knee joint in hemophilia. Orthopedic clinics of North America. 1979, 10(1): 139-173.

21) Heijnen L, and De Kleijn: Physiotherapy for the treatment of articular contractures in haemophilia. Haemophilia. 1999, 5: 16-19.

22) Cuesta-Barriuso R, Gómez-Conesa A, and López-Pina JA: Manual therapy in the treatment of ankle hemophilic arthropathy. A randomized pilot study. Physiother Theory Pract. 2014, 28: 1-6.

23) Brunner A, Stäuber F, Göhler S, Czepa D, Krüger S, Wendel M, Seuser A, and Hilberg T: Quadriceps strength, inter-extremity difference (IED) and joint status in adult persons with severe haemophilia in different age stages. Haemophilia. 2013, 19(2): 267-274.

24) Hilberg $T$, and Herbsleb M: Proprioception and isometric muscular strength in haemophilic subjects. Haemophilia. 2001, 7: 582-588.

25) Herbsleb M, and Hilberg T: Maximal isometric strength mea- sures of the quadriceps muscles. Feasibility and reliability in patients with haemophilia. Hamostaseologie. 2010, 30: S97-S103.

26) González LM, and Querol F: Force fluctuations during the maximum isometric voluntary contraction of the quadriceps femoris in haemophilic patients. Haemophilia. 2007, 13: 65-70.

27) Herbsleb M, Abou-Hamdan R, Puta C, Donath L, Kobelt R, Stanek FD, Hilberg T, and Gabriel HH: Severe and moderate haemophilia under prophylactic replacement treatment-maximal knee extensor and flexor torque of children and adolescents. Hamostaseologie. 2012, 32(1): 62-69. (in German.)

28) Pietri MM, Frontera WR, Pratts IS, and Súarez EL: Skeletal muscle function in patients with hemophilia A and unilateral hemarthrosis of the knee. Arch Phys Med Rehabil. 1992, 73(1): 22-28.

29) Voight ML, and Wieder DL: Comparative reflex response times of vastus medialis obliques and vastus lateraris in normal subjects and subjects with extensor mechanism dysfunction: an electro-myographic study. Am J Sports Med. 1991, 19: 131-137.

30) Cailliet R: Knee Pain and Disability. $3^{\text {rd }}$ Ed, Philadelphia, F.A. Davis, 1992, pp. 70-111.

31) Yamazaki H, Yokoyama H, Aoki U, Kasahara M, Ohmori Y, and Hiraki K: The knee extension muscle strength level required for independent gait: The change during the investigation period for 10 years. Kochi Rehabilitation Institute. 2002, 3: 1-6. (in Japanese)

32) Judd DL, Thomas AC, Dayton MR, and Stevens-Lapsley JE: Strength and functional deficits in individuals with hip osteoarthritis compared to healthy, olderadults. Disabil Rehabil. 2014; 36(4): 307-312.

33) McGinty G, Irrgang JJ, and Pezzullo D: Biomechanical considerations for rehabilitation of the knee. Clin Biomech. 2000, 15: 160-166

34) Kim JK, Park MG, and Shin SJ: What is the Minimum Clinically Important Difference in Grip Strength? Clin Orthop Relat Res. 2014, 472(8): 2536-2541.

35) Manco-Johnson MJ, Abshire TC, Shapiro AD, Riske B, Hacker MR, Kilcoyne R, Ingram JD, Manco-Johnson ML, Funk S, Jacobson L, Valentino LA, Hoots WK, Buchanan GR, DiMichele D, Recht M, Brown D, Leissinger C, Bleak S, Cohen A, Mathew P, Matsunaga A, Medeiros D, Nugent D, Thomas GA, Thompson AA, McRedmond K, Soucie JM, Austin H, and Evatt BL: Prophylaxis versus episodic treatment to prevent joint disease in boys with severe hemophilia. N Engl J Med. 2007, 9(6): 535-544.

36) Rodriguez-Merchan EC, Jimenez-Yuste V, Aznar JA, Hedner U, Knobe K, Lee CA, Ljung R, Querol F, Santagostino E, Valentino LA, and Caffarini A: Joint protection in haemophilia. Haemophilia. 2011, 17(2): 1-23.

37) Santavirta N, Solovieva S, Helkama O, Lehto S, Konttinen YT, and Santavirta S: Musculoskeletal pain and functional ability in haemophilia A and B. Physiotherapy and rehabilitation in haemophilia patients. Rheumatology International. 2001, 21: 15-19. 